\title{
The Moderating and Mediating Effects of Private Self- Consciousness and Self-Compassion on the Relationship between Subjective Health Perception and Mental Fitness
}

\author{
Gi-Gyung Kim¹, Sela Lim² \\ ${ }^{1}$ Student, Department of Society of Ethical Culture,Dongguk University, South Korea, \\ cgk2002@daum.net \\ ${ }^{2}$ Professor, Department of Clinical Psychology, NungIn University, South Korea, sera325@nate.com \\ Corresponding author: Sela Lim
}

\begin{abstract}
The purpose of this study is to explore the subjective health perception and mental fitness to prove the moderating model and pathway of mediating effects of negative factor of self-compassion, positive factor and private self-consciousness. The study was conducted among five operation agency cities in South Korea.A set of measures, consisting of the Subjective Health State Scale(SHSS), the Scale for Dispositional Self focused Attention(SDSAS), the Korean version of Self-Compassion Scale (K-SCS), and the Mental Fitness Scale(MFS), was administered to 562 adults. To verify the research hypothesis, M-Plus 6.12 programs were used for bootstrapping methods. In the current study, Cronbach's alpha was calculated as .948 for the Subjective Health State Scale, .927 for the Scale for Dispositional Self focused Attention, .863 for the Korean version of Self-Compassion Scale, and .978 for the Mental Fitness Scale, respectively. For the research result, this study presented descriptive statistics for each variable of respondents' and conducted correlation analysis among subjective health perception to mental fitness through negative factor of self-compassion and positive factor of selfcompassion and private self-consciousnes. Therefore, the moderating model and pathway of mediating effects on the relationship between subjective health perception, mental fitness, negative factor of self-compassion, positive factor of self-compassion and private self-consciousnesswas verified and suggested. The paper also discussed the contribution and limitation of this study, and suggestions for further research.
\end{abstract}

Keywords: Subjective Health Perception, Private Self-Consciousness, Self-Compassion, Mental Fitness, Pathway Model

\section{Introduction}

Prior studies have repeatedly emphasized more subjective mental fitness and greater level of subjective health perception[1], private self-consciousness[2-4], and negative and positive factorsof self-compassion[5]."Therefore, we have to conceptualize the role of ethical leaders regarding psychological interventions about the mental model."

There seem to be two answers: First, "among people's mental health, they want to overcome their psychological or cognitive problems as mental is a means for modification of private selfconsciousness." 
Second,"people's subjective health perception to achieve a better understanding of self-compassion (negative factor and positive factor) for their healthy life."These two aims often cannot be clearly separated, and most adults' s probably pursue both to a certain extent. People who have undergone extensive mental training for negative factor of subjective health perception and mental fitness have shown improvements in cognitive performance in negative and positive factors of self-compassion.

Prior studies have repeatedly emphasized that the more subjective mental fitness and the greater the level of subjective health perception. Therefore, after briefly surveying previous attempts to summarize the and understand the relationship and effects of subjective health perception, mental fitness, negative and positive factors of self-compassion and private self-consciousness, this study introduce existing theoretical approaches from South Korea[2]. And Germer[5] studied for adudlts, found that negative self-compassion and positive self-compassion moderated in the relation between private self-consciousness and mental health.Despite the public's growing interest and an increasing number of studies on the impact of subjective health perception and mental fitness, there is a surprising scarcity of empirical evidence, especially that which stems from research outside the therapeutic context within negative and positive factors of self-compassion.

Numerous studies have been conducted that investigate the effects on adults in relation to their field of study, yet there is an even more surprising lack of elaborate psychological theories that make sound predictions about what to expect if one works.

This theoretical approach is frequently mirrored in the measures used in this study which include all kinds of dependent variables that are not specific to work research (such as subjective health perception and mental fitness) as well as other kinds of research.

In this article, however, we place an emphasis on empirical evidence. We are convinced that real progress in understanding the effects of subjective health perception and mental fitness cannot be made if future empirical studies are not guided by better theories.

Therefore, after briefly surveying previous attempts to summarize the and understand the relationship and effectsof subjective health perception, mental fitness, negative and positive factors of selfcompassion and private self-consciousness, we introduce existing theoretical approaches from South Korea.

However, it is perceived as positive cognition based on unconscious critical thought. It also causes psychological confusion and psychopathology.

Yim and Kim reported the results of a positive correlation between subjective health perception and mental fitness[1].

Furthermore, comparative studies on cognition types focusing on psychological intervention have reported that psychological interventions positively affect victims' mental health as well as subjective health perception and mental fitness.

In addition, in some cases, psychological interventions have positive and long-term effects compared to negative and positive factors of self-compassion.

As a result, it is found that there is a lack of research on mediating effects of subjective health perception, mental fitness, private self-sonsciousness, negative and thepositive factors of selfcompassion.

This study, therefore, aims to investigate the relationship between subjective health perception, mental fitness, private self-sonsciousness and negative and the positive factors of self-compassion. This study is based on the assumption that the issues of cognitive maladjustment due to various subjective health perception and mental fitness experienced by meditators living in South Korea are related to private self-consciousness and the negative and positive factors of self-compassion.

First, this study examines whether subjective health perception, private self-consciousness and thenegative and positive factors of self-compassion, and mental fitness have significant correlations. 
Second, the study examines whether subjective health perception, in terms of their effect on mental fitness, will have a continuous mediating effect on private self-consciousness and the negativeand positive factors of self-compassion.

This research question of this study are:

1) How perceived subjective health perception of adults affects mental fitness?

2) Does private self-consciousness and the negative and positive factors of self-compassion as a moderator between perceived subjective health and mental fitness of adults?

This study may be useful for enhancing the understanding of the factors affecting the meditator, and could provide the opportunity for clinicians to provide basic data on research and psychotherapy.

\section{Methods}

\subsection{Demographic Characteristics of Participantsand Data Collection}

This study was conducted from January 2018 to June 2019.Participants were male and female (adults) in Seoul, Korea. The data from 562 males and females (adults) in Seoul, Korea were used in the final analysis. As shown in Table 1, the ages and gender of participants were in the following:over the age of 18 (33 or 5.87\%), over the age of 31 (164 or $29.18 \%$ ), over the age of 41 (115 or $20.46 \%$ ), over the age of 51 (161 or $28.64 \%$ ), and over the age of 61 (90 or $16.01 \%)$.

[Table 1] Age and Gender of Participants(N=562)

\begin{tabular}{|c|c|c|}
\hline \multicolumn{2}{|r|}{ Age } & $\operatorname{Total}(\%)$ \\
\hline \multirow{5}{*}{ Divison } & Over the age of 18 & $33(5.87 \%)$ \\
\hline & Over the age of 31 & $164(29.18 \%)$ \\
\hline & Over the age of 41 & $115(20.46 \%)$ \\
\hline & Over the age of51 & $161(28.64 \%)$ \\
\hline & Over the age of61 & $90(16.01 \%)$ \\
\hline \multicolumn{2}{|r|}{ Total } & $562(100.00 \%)$ \\
\hline
\end{tabular}

\subsection{Tools}

\subsubsection{Subjective Health Scale}

In order to measure the subjective health towards their chosen major, this study used Speak, CowartandPellet's[6] tool developed by Yim and Kim[1]. This tool consists of a total of questions. The measurement criterion uses a five-point Likert scale with one point as 'not at all' to five points as 'very much'. The higher the score, the higher the degree of satisfaction towards their major. As for the reliability of the tool, the Cronbach $\alpha$ value in Lee's study[7] was .891, and theCronbach $\alpha$ value in this study was .948 .

\subsubsection{Self Compassion Scale}

In order to measure the self compassiontowards their chosen major, this study used Neff's[8] tool developed by Gu and Jung[9]. This tool consists of a total of 26 questions. The measurement criterion uses a five-point Likert scale with one point as 'not at all' to five points as 'very much'. The higher the score, the higher the degree of satisfaction towards their major. As for the reliability of the tool, the Cronbach $\alpha$ value in Gu and Jung's study was.82.Positive factors and negative factors were measured individually; Cronbach's a .795 on positive factors, and Cronbach's a .706 on negative factors, and a total of .863 Cronbach's a. 


\subsubsection{Self-focused AttentionScale}

In order to measure the self-focused attention towards their chosen major, this study used Fenigstein's[10]tool developed by Lee and Kwon[11]. This tool consists of a total of 30 questions. The measurement criterion uses a five-point Likert scale with one point as 'not at all' to five points as 'very much'. The higher the score, the higher the degree of satisfaction towards their major. As for the reliability of the tool, the Cronbach $\alpha$ value in Lee and Kwon's study[11] was .93, and the Cronbach $\alpha$ value in this study was .927 .

\subsubsection{Mental Fitness Scale}

In order to measure the mental fitness towards their chosen major, this study used Cho, Woo, Kim, Byean, Kang, Choi, Bang, Lee,Bark, Chae, Yim and Cho's[12] tool. This tool consists of a total of 20 questions. The measurement criterion uses a four-point Likert scale with one point as 'not at all' to fourpoints as 'very much'. The higher the score, the higher the degree of satisfaction towards their major. As for the reliability of the tool, the Cronbach $\alpha$ value in Cho's study[12] was .901, and the Cronbach $\alpha$ value in this study was .978 .

\subsection{Data analysis}

The datacollected for this study was analyzed using the IBM SPSS V.21 and M-Plus 6.12as follows[13]:We used a 95\% confidence interval based on the empirical distribution estimated by bootstrapping to verify the mediating effect. Bootstrapping interpreted the sample as statistically significant if it did not know the distribution of the population and if the 95\% confidence interval did not include zero[14].

\section{Results}

\subsection{Correlation analysis}

\subsubsection{Correlation analysis of variable}

The results of Pearson's correlation analysis were analyzed to discover the relationship between subjectivehealth perception and private self-consciousness and negative and positive factors of selfcompassion and mental fitness. There was significant correlation between subjectivehealth perception and private self-consciousness and negative and positive factors of self-compassion and mental fitness. However, there were significant negative correlationsbetween subjectivehealth perception and private self-consciousness $(r=-.675, \mathrm{p}<.01)$, the negative factor of self-compassion $(r=-.714, p<.01)$.

Also, there were significant positivecorrelationsbetweensubjectivehealth perception and positive factorof self-compassion( $(\mathrm{r}=.609, \mathrm{p}<.01)$, and mental fitness( $\mathrm{r}=.139, \mathrm{p}<.01)$. The was asignificantpositivecorrelationbetween private self-consciousness and negative factor of selfcompassion $(\mathrm{r}=.870, \mathrm{p}<.01)$ a significant negative pogitivecorrelationbetweenprivate selfconsciousness and positive factor of self-compassion $(r=-.706, p<.01)$. a significant negative correlationbetweenprivate self-consciousness and mental fitness $(r=-.510, p<.01)$. a significant negative correlation between negative factor of self-compassion and positive factor of selfcompassion( $\mathrm{r}=-.802, \mathrm{p}<.01)$.a significant negative correlationbetweennegative factor of selfcompassion and mental fitness $(\mathrm{r}=-.539, \mathrm{p}<.01)$. a significant positive correlationbetweenpositive factor of self-compassion and mental fitness $(r=.276, \mathrm{p}<.01)$. 
[Table 2] Correlation Analysis of Variable

\begin{tabular}{|c|c|c|c|c|c|}
\hline & $\begin{array}{l}\text { Subjective } \\
\text { Health } \\
\text { Perception }\end{array}$ & $\begin{array}{c}\text { Private Self- } \\
\text { Consciousness }\end{array}$ & $\begin{array}{l}\text { Negative factor of } \\
\text { Self-Compassion }\end{array}$ & $\begin{array}{c}\text { Positive factor } \\
\text { of Self-Compassion }\end{array}$ & Mental Fitness \\
\hline $\begin{array}{l}\text { Subjective } \\
\text { Health } \\
\text { Perception }\end{array}$ & 1 & & & & \\
\hline $\begin{array}{l}\text { Private Self- } \\
\text { Consciousness }\end{array}$ & $-.675^{* *}$ & 1 & & & \\
\hline $\begin{array}{c}\text { Negative } \\
\text { factor of Self- } \\
\text { Compassion }\end{array}$ & $-.714 * *$ & $.870^{\text {*** }}$ & 1 & & \\
\hline $\begin{array}{l}\text { Positive factor } \\
\text { of Self- } \\
\text { Compassion }\end{array}$ & $.609 * *$ & $-.706 * *$ & $-.802 * *$ & 1 & \\
\hline Mental Fitness & $.139 * *$ & $-.510 * *$ & $-.539 * *$ & $.276^{* *}$ & 1 \\
\hline
\end{tabular}

\subsubsection{Correlation Analysis of Variable of Subfactor}

The results of Pearson's correlation analysis were analyzed to discover the relationship between sub-factors of subjectivehealth perception and private self-consciousness and self-compassion and mental fitness.

There were significant negative correlations between subjectivehealthperceptionandgeneral selffocused attentionofself-consciousness $(\mathrm{r}=-.383, \mathrm{p}<.01)$.

Also, there were significant negative correlations between subjectivehealth perception and defensive self-focused attention of private self-consciousness $(r=-.478, \mathrm{p}<.01)$. a significant negative correlation betweensubjectivehealth perception and negative factor of self-compassion ( $\mathrm{r}=-.714$, $\mathrm{p}<.01)$.a significant negative correlation betweensubjectivehealth perception and positive factor of self-compassion $(\mathrm{r}=.609, \mathrm{p}<.01)$. a significant positivecorrelation betweensubjectivehealth perception and mental energy of mental fitness $(\mathrm{r}=.401, \mathrm{p}<.01) . \mathrm{a}$ significant positive correlation betweensubjectivehealth perception and empathic communication of mental fitness $(\mathrm{r}=.324, \mathrm{p}<.01)$.a significant negative correlation betweensubjectivehealth perception and flexibility of mental fitness( $\mathrm{r}=-.125, \mathrm{p}<.01)$.a significant negative correlation betweensubjectivehealth perception and selfassurance of mental fitness $(\mathrm{r}=-.129, \mathrm{p}<.01)$. There was a significant negative correlation between general self-focused attention of private self-consciousness and negative factor of self-compassion $(\mathrm{r}=.151, \mathrm{p}<.01)$. a significant negative correlation between general self-focused attention of private self-consciousness and positive factor of self-compassion $(\mathrm{r}=-.402, \mathrm{p}<.01)$.a no significant correlation betweengeneral self-focused attention of private self-consciousnessandmental energy and empathic communication of mental fitness. a significant positivecorrelation betweengeneral self-focused attention of private self-consciousness andof flexibility mental fitness $(r=.336, p<.01)$.a significant negative correlation betweengeneral self-focused attention of private self-consciousnessandof selfassurance of mental fitness $(\mathrm{r}=-.256, \mathrm{p}<.01)$. There was a significant positive correlation between defensive self-focused attention of private self-consciousness andnegative factor of selfcompassion( $\mathrm{r}=.782, \mathrm{p}<.01)$, a significant negaitive correlation between defensive self-focused attention of private self-consciousness andpositive factor of self-compassion( $(r=-.499, \quad p<.01) . a$ significant negaitive correlation between defensive self-focused attention of private self-consciousness andmental energy of mental fitness $(\mathrm{r}=-.660, \mathrm{p}<.01)$. a significant negaitive correlation between defensive self-focused attention of private self-consciousness and empathic communication of mental fitness( $r=-.551, \mathrm{p}<.01)$. asignificant negaitive correlation between defensive self-focused attention of private self-consciousness and flexibility of mental fitness(r=-.477, $\mathrm{p}<.01)$. asignificant 
negaitivecorrelation between defensive self-focused attention of private self-consciousness and selfassurance of mental fitness( $\mathrm{r}=-.171, \mathrm{p}<.01)$. There was a significant negative correlation between negative factor of self-compassion and mental energy of mental fitness(r=-.745, $\mathrm{p}<.01)$. asignificant negative correlation between negative factor of self-compassion and empathic communication of mental fitness( $\mathrm{r}=-.671, \mathrm{p}<.01)$.asignificant negative correlation between negative factor of selfcompassion and flexibility of mental fitness $(\mathrm{r}=-.275, \mathrm{p}<.01)$. asignificant negative correlation between negative factor of self-compassion and self-assurance of mental fitness(r=-.177, $\mathrm{p}<.01)$. There was a significant positive correlation between positive factor of self-compassion and mental energy of mental fitness $(r=.481, \mathrm{p}<.01)$. asignificant pogitive correlation between positive factor of selfcompassion and empathic communication of mental fitness $(\mathrm{r}=.438, \mathrm{p}<.01)$. But,there was no significant between positive factor of self-compassion and flexibility and self-assurance of mental fitness.

[Table 3] Correlation Analysis of Variable of Subfactor

\begin{tabular}{|c|c|c|c|c|c|c|c|c|c|c|}
\hline & \multirow[b]{2}{*}{$\begin{array}{c}\text { Subjecti } \\
\text { ve } \\
\text { Health } \\
\text { Percepti } \\
\text { on }\end{array}$} & \multicolumn{2}{|c|}{$\begin{array}{c}\text { Private Self- } \\
\text { Consciousness }\end{array}$} & \multicolumn{2}{|c|}{ Self-Compassion } & \multicolumn{4}{|c|}{ Mental Fitness } \\
\hline & & & $\begin{array}{l}\text { Genera } \\
\text { 1 Self- } \\
\text { focuse } \\
\text { d } \\
\text { Attenti } \\
\text { on }\end{array}$ & $\begin{array}{c}\text { Self- } \\
\text { Absorpti } \\
\text { on }\end{array}$ & $\begin{array}{l}\text { Negative } \\
\text { factor }\end{array}$ & $\begin{array}{l}\text { Positive } \\
\text { factor }\end{array}$ & $\begin{array}{c}\text { Menta } \\
1 \\
\text { energy }\end{array}$ & $\begin{array}{l}\text { Socail } \\
\text { sense }\end{array}$ & $\begin{array}{c}\text { Flexibil } \\
\text { ity }\end{array}$ & $\begin{array}{l}\text { Me } \\
\text { ntal } \\
\text { stre } \\
\text { ngh }\end{array}$ \\
\hline \multicolumn{2}{|c|}{$\begin{array}{c}\text { Private Self- } \\
\text { Consciousness }\end{array}$} & 1 & & & & & & & & \\
\hline \multirow{2}{*}{$\begin{array}{l}\text { Private } \\
\text { Self- } \\
\text { Conscious } \\
\text { ness }\end{array}$} & $\begin{array}{l}\text { General } \\
\text { Self- } \\
\text { focused } \\
\text { Attention }\end{array}$ & $-.383^{* *}$ & 1 & & & & & & & \\
\hline & $\begin{array}{c}\text { Self- } \\
\text { Absorption }\end{array}$ & $-.478^{* *}$ & $-.277^{* *}$ & 1 & & & & & & \\
\hline \multirow{2}{*}{$\begin{array}{c}\text { Self- } \\
\text { Compassi } \\
\text { on }\end{array}$} & $\begin{array}{l}\text { Negative } \\
\text { factor }\end{array}$ & $-.714^{* *}$ & $.151^{* *}$ & $.782^{* *}$ & 1 & & & & & \\
\hline & $\begin{array}{l}\text { Positive } \\
\text { factor }\end{array}$ & $.609^{* *}$ & $-.402^{* *}$ & $-.499^{* *}$ & $-.802^{* * *}$ & 1 & & & & \\
\hline \multirow{4}{*}{$\begin{array}{l}\text { Mental } \\
\text { Fitness }\end{array}$} & $\begin{array}{l}\text { Mental } \\
\text { energy }\end{array}$ & $.401^{* *}$ & .034 & $-.660^{* *}$ & $-.745^{* *}$ & $.481^{* *}$ & 1 & & & \\
\hline & $\begin{array}{l}\text { Socail } \\
\text { sense }\end{array}$ & $.324^{* *}$ & .053 & $-.551^{* *}$ & $-.671^{* *}$ & $.438^{* *}$ & $\begin{array}{c}.955^{*} \\
* \\
\end{array}$ & 1 & & \\
\hline & Flexibility & $-.125^{* *}$ & $.366^{* *}$ & $-.477^{* *}$ & $-.275^{* *}$ & -.033 & $\begin{array}{c}.726^{*} \\
*\end{array}$ & $\begin{array}{c}.762^{*} \\
*\end{array}$ & 1 & \\
\hline & $\begin{array}{l}\text { Mental } \\
\text { strengh }\end{array}$ & $-.129^{* *}$ & $-.256^{* *}$ & $-.171^{* *}$ & $-.177^{* *}$ & .079 & $\begin{array}{c}.500^{*} \\
*\end{array}$ & $\begin{array}{c}.474^{*} \\
* \\
\end{array}$ & $.698^{* *}$ & 1 \\
\hline
\end{tabular}

\subsection{ModeratingModel}

We performed bootstrapping by extracting 2000 parts of the escapism of subjective health perception to the mental fitness through the negative and positive factors of self-compassion. As a result, the indirect effect was significant because the path through negative factor of self$\operatorname{compassion}(\beta=.190$, C.I[.145 .249]), the positive factor of self-compassion( $\beta=-.124$, C.I[-.160 -.096]), private self-sonsciousness( $\beta=.166$, C.I[.110 .237]), private self-consciousness and negative factor of self-compassion( $\beta=.245$, C.I[.214 .300]), and the private self-consciousness and positive factor of 
self-compassion( $\beta=-.124$, C.I[-.160 -.096]) all not included 0 in the $95 \%$ confidence interval of the indirect effect.

[Table 4]Moderating Model

\begin{tabular}{|c|c|c|c|c|c|c|c|c|c|}
\hline \multicolumn{7}{|c|}{ Pathway } & \multirow{2}{*}{$\begin{array}{c}\text { Total effect } \\
\text { [bootstrappin } \\
\mathrm{g} \\
\text { CI 95\%] }\end{array}$} & \multirow{2}{*}{$\begin{array}{c}\text { Total indirect } \\
\text { effect } \\
\text { [bootstrappin } \\
\mathrm{g} \\
\text { CI 95\%] }\end{array}$} & \multirow{2}{*}{$\begin{array}{c}\text { Indirect effect } \\
\text { [bootstrappin } \\
\mathrm{g} \\
\text { CI } 95 \% \text { ] }\end{array}$} \\
\hline $\begin{array}{l}\text { Independen } \\
\text { t variable }\end{array}$ & & $\begin{array}{l}\text { Parameter } \\
\text { (a) }\end{array}$ & & $\begin{array}{l}\text { Parameter } \\
\text { (b) }\end{array}$ & & $\begin{array}{l}\text { Dependen } \\
\text { t variable }\end{array}$ & & & \\
\hline $\begin{array}{l}\text { Subjective } \\
\text { Health } \\
\text { Perception }\end{array}$ & & $\rightarrow$ & & $\begin{array}{c}\text { Negative } \\
\text { factor of } \\
\text { Self- } \\
\text { Compassio } \\
\text { n }\end{array}$ & $\rightarrow$ & $\begin{array}{l}\text { Mental } \\
\text { Fitness }\end{array}$ & \multirow{5}{*}{$\begin{array}{c}.353 \\
{[.303 \sim .400]}\end{array}$} & \multirow{5}{*}{$\begin{array}{c}.353 \\
{[.303 \sim .400]}\end{array}$} & $\begin{array}{c}.190 \\
{[.145 \sim .249]}\end{array}$ \\
\hline $\begin{array}{l}\text { Subjective } \\
\text { Health } \\
\text { Perception }\end{array}$ & & $\rightarrow$ & & $\begin{array}{c}\text { Positive } \\
\text { factor of } \\
\text { Self- } \\
\text { Compassio } \\
\text { n }\end{array}$ & $\rightarrow$ & $\begin{array}{l}\text { Mental } \\
\text { Fitness }\end{array}$ & & & $\begin{array}{c}-.133 \\
{[-.180-.090]}\end{array}$ \\
\hline $\begin{array}{l}\text { Subjective } \\
\text { Health } \\
\text { Perception }\end{array}$ & $\rightarrow$ & $\begin{array}{c}\text { Private Self- } \\
\text { Consciousnes } \\
\mathrm{s}\end{array}$ & & $\rightarrow$ & & $\begin{array}{l}\text { Mental } \\
\text { Fitness }\end{array}$ & & & $\begin{array}{c}.166 \\
{[.110 \sim .237]}\end{array}$ \\
\hline $\begin{array}{l}\text { Subjective } \\
\text { Health } \\
\text { Perception }\end{array}$ & $\rightarrow$ & $\begin{array}{c}\text { Private Self- } \\
\text { Consciousnes } \\
\text { s }\end{array}$ & $\rightarrow$ & $\begin{array}{c}\begin{array}{c}\text { Negative } \\
\text { factor of } \\
\text { Self- } \\
\text { Compassio } \\
\text { n }\end{array} \\
\end{array}$ & $\rightarrow$ & $\begin{array}{l}\text { Mental } \\
\text { Fitness }\end{array}$ & & & $\begin{array}{c}.245 \\
{[.214 \sim 300]}\end{array}$ \\
\hline $\begin{array}{l}\text { Subjective } \\
\text { Health } \\
\text { Perception }\end{array}$ & $\rightarrow$ & $\begin{array}{l}\text { Private Self- } \\
\text { Consciousnes } \\
\text { s }\end{array}$ & $\rightarrow$ & $\begin{array}{c}\text { Positive } \\
\text { factor of } \\
\text { Self- } \\
\text { Compassio } \\
\text { n }\end{array}$ & $\rightarrow$ & $\begin{array}{l}\text { Mental } \\
\text { Fitness }\end{array}$ & & & $\begin{array}{c}-.124 \\
{[-.160-.096]}\end{array}$ \\
\hline
\end{tabular}

\subsection{Pathway ofMediatingEffects}

A subjectivehealth perceptionanalysis was conducted to confirm the effects ofmental fitness. Specifically, subjectivehealth perception and mental fitness had a significant effect on negative positive factor of self-compassion. In addition, subjective health perception were found to have a significant effect on mental fitness. In addition, subjectivehealth perception were found to have a significant negative effect on private self-consciousness $(\beta=-.660, p<.001)$ and negative self$\operatorname{compassion}(\beta=-.307, \mathrm{p}<.001)$. Private self-sonsciousnesswere found to have a significant positive effect on negative self-compassion $(\beta=.623, \mathrm{p}<.001)$, a significant negative effect on positive self$\operatorname{compassion}(\beta=-.441, \mathrm{p}<.001)$, and a significant negative effect on mental fitness $(\beta=-.251, \mathrm{p}<.001)$. Negative self-compassion were found to have a significant negative effect on mental fitness $(\beta=-.619$, $\mathrm{p}<.001)$. Lastly, positive self-compassion were found to have a significant negative effect on mental fitness $(\beta=-.425, \mathrm{p}<.001)$.

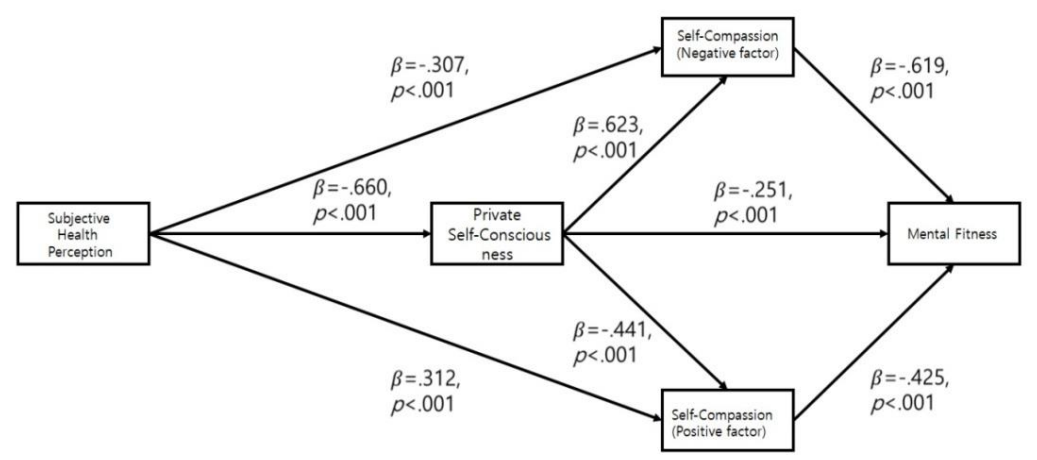

[Fig. 1] Pathway ofMediating Effects 
[Table 5]PathwayofMediatingEffects

\begin{tabular}{|c|c|c|c|c|c|c|c|c|}
\hline \multicolumn{3}{|c|}{ Pathway of Mediating Effects } & \multirow{2}{*}{$\begin{array}{c}\text { B } \\
-0.299\end{array}$} & \multirow{2}{*}{$\begin{array}{c}\mathrm{B} \\
-660\end{array}$} & \multirow{2}{*}{$\begin{array}{l}\text { s.e } \\
0.024\end{array}$} & \multicolumn{2}{|c|}{$\mathrm{t}$} & \multirow{2}{*}{$\begin{array}{l}\mathrm{p} \\
.000\end{array}$} \\
\hline $\begin{array}{c}\text { Subjective } \\
\text { Health Perception }\end{array}$ & $\rightarrow$ & $\begin{array}{c}\text { Private } \\
\text { Self- } \\
\text { Consciousness }\end{array}$ & & & & -27.874 & *** & \\
\hline $\begin{array}{c}\text { Subjective } \\
\text { Health Perception }\end{array}$ & $\rightarrow$ & \multirow{2}{*}{$\begin{array}{c}\text { Negative factor } \\
\text { of } \\
\text { Self- } \\
\text { Compassion }\end{array}$} & -0.134 & -.307 & 0.029 & -10.731 & $* * *$ & .000 \\
\hline $\begin{array}{c}\text { Private } \\
\text { Self- } \\
\text { Consciousness } \\
\end{array}$ & $\rightarrow$ & & 0.601 & .623 & 0.026 & 23.836 & $* * *$ & .000 \\
\hline $\begin{array}{c}\text { Subjective } \\
\text { Health Perception }\end{array}$ & $\rightarrow$ & \multirow{2}{*}{$\begin{array}{l}\text { Positive factor } \\
\text { of } \\
\text { Self- } \\
\text { Compassion }\end{array}$} & 0.153 & .312 & 0.040 & 7.879 & $* * *$ & .000 \\
\hline $\begin{array}{c}\text { Private } \\
\text { Self- } \\
\text { Consciousness } \\
\end{array}$ & $\rightarrow$ & & -0.478 & -.441 & 0.038 & -11.474 & $* * *$ & .000 \\
\hline $\begin{array}{c}\text { Private } \\
\text { Self- } \\
\text { Consciousness }\end{array}$ & $\rightarrow$ & \multirow{3}{*}{ Mental Fitness } & -0.591 & -.251 & 0.057 & -4.408 & $* * *$ & .000 \\
\hline $\begin{array}{c}\text { Negativefactor } \\
\text { of } \\
\text { Self-Compassion }\end{array}$ & $\rightarrow$ & & -1.511 & -.619 & 0.063 & -9.832 & $* * *$ & .000 \\
\hline $\begin{array}{c}\text { Positivefactor } \\
\text { of } \\
\text { Self-Compassion }\end{array}$ & $\rightarrow$ & & -0.926 & -.425 & 0.048 & -8.828 & $* * *$ & .000 \\
\hline
\end{tabular}

\section{Conclusions}

The research result, this study presented descriptive statistics for each variable of respondents' and conducted correlation analysis among subjective health perception to mental fitness through negative factor of self-compassion and positive factor of self-compassion and private self-consciousnes. Therefore, the moderating model and pathway of mediating effects on the relationship between subjective health perception, mental fitness, negative factor of self-compassion, positive factor of selfcompassion and private self-consciousnesswas verified and suggested..

Clark \&Wells[1] and Yim\& Kim[2] discovered that most causes of objective health perception that interfere with mental fitness are experienced in people's life.

Silvia Eichstaedt andPhilips[3] discovered that most causes of private self-consciousness that interfere with mental fitness are experienced in adults'smental health.

Greenberg[4] discovered that most causes of private self-consciousness that interfere with mental fitness are experienced in clinets's mental health.

Germer[5] discovered among mindful experienced people that most of the causes of negative selfcompassion and positive self-compassion that interfere with thinking are related to mental fitness.

Kim[15]discovered among adult that most of the causes ofobjective health perception, private selfconsciousness, negative self-compassion and positiveself-compassion that interfere with thinking are related to mental fitness.

Therefore, objective health perception of changes in these cognition conditions could lead to a deterioration of psychological function.In conclusion, the mediating effect of objective health perception, mental fitness, negative self-compassion and positive self-compassion of adults was solely analyzed in South Korea, and significant results were obtained.

This study is significant because it collected data from adults of Korean, a large corporation, to establish a path model and record correlations among subjective health perception, mental fitness, negative self-compassion and positive self-compassion and verify these findings through a continuous 
mediation model analysis.

Second, it verified the mediating effects of negative self-compassion and positiveself-compassion on the relationship between subjective health perception and mental fitness.

Through this, it was confirmed that subjective health perception influence mental fitness through job ethics and job engagement. Third, it verified the significance of the indirect effect of job ethics and job engagement on the relationship between subjective health perception and mental fitness. Through this, it was confirmed that subjective health perception influenced mental fitness through negative selfcompassion and positive self-compassion.

The limitations of this study and suggestions for follow-up studies are as follows. First, it was expected that the Korean adults included in the study showed social desirability in the questionnaire since they knew that the researcher of this study was a main executive of their place. Thus, the sincerity of their answers cannot be verified.

Second, the study found that negative self-compassion and positive self-compassion acted as mediating factors in subjective health perception affecting mental fitness, but failed to discover a structural equation model, which entails a more detailed research method.

Third, a mediation model consistent with the results of the study could not be found among domestic and foreign research data. A research model was constructed based on configurations presented in a prior study showing the correlation between each variable. However, there was no previous research on a moderating model and pathwayof mediatingeffects that combined subjective health perception, mental fitness, negative self-compassion and positive self-compassion for employees at domestic or foreign pharmaceutical companies. Thus, there was a limitation in discussing the significance of the results as it failed to reflect the results of previous studies with consistent results.

Fourth, this study did not construct a program reflecting subjective health perception , mental fitness, negative self-compassion and positive self-compassion. Instead, it merely set up a study model by simply reflecting the correlation results of previous studies and the results were not confirmed through experimental groups.

The suggestions for follow-up research based on the results of this study are as follows.

First, this study showed significant correlations between subjective health perception , mental fitness, negative self-compassion and positive self-compassion. This indicates that mental fitness levels may be lowered with appropriate subjective health perception factors. It also indicates that changes to negative self-compassion and positive self-compassion can be elicited as well.

Nevertheless, it is important to note that results from this study could be used in reference to cognitive work style, especially during counseling interventions and in clinical settings.

In follow-up studies, it is necessary to measure the subjective health perception, mental fitness, negative self-compassion and positiveself-compassion of various subjects, not just the adults, in order to clearly compare and verify their relationships with the variables used in this study.

Second, studies on subjective health perception, mental fitness, negative self-compassion and positiveself-compassion in Korea are insufficient. Most studies only measured subjective health perception, mental fitness, negative self-compassion and positiveself-compassion independently. Moreover, there have been no studies on how subjective health perception lead to mental fitness throughnegative self-compassion and positiveself-compassion, and noneverified this through quantitative measurements. Therefore, it is necessary to repeatedly verify whether the derived moderating model and pathway of mediatingeffects is appropriate within the scope of variables such as subjective health perception, mental fitness, negative self-compassion and positiveself-compassion .

Third, this study used a moderating model and pathway of mediatingeffects to validate the results of the continuous mediating model. A moderating model and pathwayof mediatingeffectsis a good quantitative research method that reduces errors and finds a hypothesis that is connected to the real world. In order to supplement this study, it is necessary to conduct more detailed research through 
structural equation model validation in order to clarify the relationship between each element constituting the model or the relationships between each element and the whole through statistical techniques.

Fourth, more research methods should be considered, such as experimental studies, observation methods, interview methods, or longitudinal studies rather than cross-sectional studies.

Lastly, in the follow-up studies, it is necessary to develop a program that can be applied to Korean adultsand verify its effectiveness by reflecting the path model in this study.

\section{References}

[1] D. M. Clark, A. Wells, A cognitive model of social Phobia, Social Phobia: Diagnosis, Assessment, and Treatment, UK: The Guilford Press (1995).

[2] Y. H. Yim, J. D. Kim, The Pathway Model of Physical Health Perception on Mental Health: Mediating Effect of Selfcompassion, Private Self-consciousness, and Self Critical Cognition, The Journal of Humanities and Social Sciences 21, (2019), Vol.10, No.1, pp.411-423.

[3] P. J. Silvia, J. Eichstaedt, A. G. Philips, Are rumination and eflection types of self-focused attention?, Personality and Iindividual Differences, (2005), Vol.38, No.4, pp.871-881.

[4] L. S. Greenberg, Emotion-focused therapy: Coaching clinets to work through their feelings, USA Washington, BC: American Psychological Association, (2002)

[5]C. K. Germer, The mindful path to self-compassion: Feeling yourself from destructive houghts and emotions, UK: The Guilford Press, (2009)

[6] D. L. Speake, M. E. Cowart, K. Pellet, Health perception and life styles of the elderly, Research in Nursing \& Health, (1989), Vol.12, No.2, pp.93-100. DOI: 10.1002/nur.4770120206

[7] M. D. Lee, The Effects of the Physical and Emotional Activities on the Health, Self Perceived Health Status and the Depression of the Elderly, Master's Thesis, Gachon University, (2012)

[8] K. D. Neff, The Development and validation of a scale to measure self-compassion, Self and Identity, (2003), Vol.2, No.3, pp.223-250.

[9] D. Y, Gu, M. C. Jung, Study of Factor Validity of Korean Version Self-Compassion, Journal of the Korean Data Analysis Society, (2016), Vol.18, No.3(B), pp.1595-1610.

[10] A. Fenigstein, M. F. Scheier, A. H. Buss, Public and private self-consciousness: Assessment and theory, Journal of Consulting and Clinical Psychology, (1975), Vol.43, No.4, pp.522-527.

[11] J. Y. Lee, S. M. Kwon, Development of the Scale for Dispositional Self-focused Attention in Social situation, The Korean Journal of Clinical Psychology in Clinical Psychology, (2005), Vol.24, No.2, pp 451-464

[12]S. H. Cho, J. M. Woo, W. Kim, K. R. Byean, E. H. Kang, S. U. Choi, S. Y. Bang, S. H. Lee, Y. M. Bark, J. H. Chae, S. K. Yim, K. S. Cho, The Development of the 'Mental Fitness' Scale, Neuro psychiatric Association, (2011), Vol.50, No.2, pp.116-124.

[13] L. K. Muthén, B. O. Muthén, Mplus User's Guide, Muthén and Muthén, (2011), https://www.statmodel.com/html_ug.shtml

[14] P. E. Shrout, N. Bolger, Mediation in experimental and non experimental studies: new procedures and recommendations, Psychological methods, (2002), Vol.7, No.4, p.422.

[15] J. K. Kim, The Pathway model of Subjective Health Perception to Mental Fitness through Private SelfConsciousness and Self-Compassion, Master's Thesis, Nungin Graduate School of University, (2017) 